First principles based simulations of instabilities and turbulence

This article has been downloaded from IOPscience. Please scroll down to see the full text article.

2004 Plasma Phys. Control. Fusion 46 B51

(http://iopscience.iop.org/0741-3335/46/12B/005)

The Table of Contents and more related content is available

Download details:

IP Address: 128.178.125.35

The article was downloaded on 08/04/2010 at 16:31

Please note that terms and conditions apply. 


\title{
First principles based simulations of instabilities and turbulence
}

\author{
L Villard $^{1}$, P Angelino ${ }^{1}$, A Bottino ${ }^{1}$, S J Allfrey ${ }^{1}$, R Hatzky ${ }^{2}$, Y Idomura ${ }^{3}$, \\ O Sauter ${ }^{1}$ and T M Tran ${ }^{1}$ \\ ${ }^{1}$ Centre de Recherches en Physique des Plasmas, Ecole Polytechnique Fédérale de Lausanne, \\ Association Euratom-Suisse, Lausanne, Switzerland \\ ${ }^{2}$ Rechenzentrum der Max-Planck Gesellschaft, Garching, Germany \\ 3 Japan Atomic Energy Research Institute, Naka, Japan \\ E-mail: laurent.villard@epfl.ch
}

Received 2 July 2004

Published 17 November 2004

Online at stacks.iop.org/PPCF/46/B51

doi:10.1088/0741-3335/46/12B/005

\begin{abstract}
It is now widely believed that low frequency turbulence developing from smallscale instabilities is responsible for the phenomenon of anomalous transport generally observed in magnetic confinement fusion experiments. The microinstabilities are driven by gradients of equilibrium density, ion and electron temperatures and magnetic field strength. Gyrokinetic theory is based on the Vlasov-Maxwell equations and, consistent with the ordering, averages out the fast particle gyromotion, reducing the phase space from 6 to 5 dimensions. Solving the resulting equations is a non-trivial task. Difficulties are associated with the magnetic confinement geometry, the strong disparities in space and time scales perpendicular and parallel to $\boldsymbol{B}$, the different time scales of ion and electron dynamics, and the complex nonlinear behaviour of the system. The main numerical methods are briefly presented together with some recent developments and improvements to the basic algorithms. Recent results are shown, with emphasis on the roles of zonal $E \times B$ flows, of parallel nonlinearity and of toroidal coupling on the saturation of ion temperature gradient (ITG) driven turbulence in tokamaks.
\end{abstract}

\section{Introduction}

Instabilities and turbulence are ubiquitous phenomena in plasmas. There is great interest in these phenomena, especially in the context of magnetic confinement fusion research, which has lasted for several decades and is still growing. The understanding of anomalous transport has greatly improved over the past years, thanks in large part to the role of large-scale numerical simulations [1-16]. Predictions used for the design of the next generation of experimental 
fusion reactors have been largely made on extrapolations from a range of existing experiments worldwide and assumed semi-empirical scaling laws. However, a full comprehension of the basic physical processes underlying the confinement properties is still missing.

Instabilities can be divided into two types: the macro-instabilities, defined as MHD instabilities developing on a length scale comparable to the device size, and the microinstabilities, which have a typical scale of the same order as the Larmor radius. Computational tools for MHD problems in tokamak (axisymmetric) geometry were developed more than 20 years ago and have been extremely useful in determining the operational limits in tokamaks. More sophisticated numerical tools are still being developed to tackle more difficult problems. Examples of such problems are: the instabilities in the presence of a magnetic separatrix [17]; the role of internal modes as triggers of sawtooth relaxations [18]; stability analysis and bootstrap current optimization for more exotic shapes (low aspect ratio [19], fully threedimensional configurations [20]); and the linear and nonlinear threshold of neoclassical tearing modes (NTMs) [21]. Also, an important class of modes has been shown to be destabilized in the presence of a fast (non-thermal) ion population: the Alfvén eigenmodes (AE), and in particular its toroidicity induced branch [22-25].

This paper focuses on micro-instabilities and their nonlinear evolution. Equilibrium gradients of temperature, density or magnetic field strength are the driving source of these instabilities. Unstable modes lead to a nonlinear state with a finite fluctuation amplitude. This is accompanied by the generation of an anomalous heat and/or particle flux across magnetic field lines, typically much larger than the transport calculated from collisional behaviour (classical or neoclassical). Theory has focused in particular on low frequency modes (as compared to the cyclotron frequency of plasma species). Among the different types of micro-instabilities, the ion temperature gradient (ITG) mode [26] has received particular attention. The computed growth rates, $\gamma$, and perpendicular wavenumbers, $k_{\perp}$, are such that a mixing length estimate of the diffusion coefficient, $\gamma / k_{\perp}^{2}$, typically gives values that are comparable to those needed to explain various confinement experiments. ITG modes (and other modes such as the trapped electron mode (TEM) [27], and the electron temperature gradient (ETG) mode [28, 29]) have a finite temperature gradient length threshold, above which the growth rate is seen to increase strongly: thus the system tends to react to an increased input power by only a slight increase in the temperature gradient while the heat flux is strongly increased. This is (at least qualitatively) in agreement with the degradation of confinement time with input power and the profile stiffness observed in many experiments. The low frequency character of the fluctuations under study is at the basis of the gyrokinetic ordering, in which the Vlasov-Maxwell equations are averaged over the particle gyromotion, thus reducing the phase space of the distribution function from 6 to 5 dimensions.

Studying linear behaviour alone is not enough to understand the many physical mechanisms involved. Research in the past decade has focused on the processes responsible for the saturation of fluctuations to a finite amplitude. In particular, the observation of 'transport barriers' [30], regions in which the anomalous transport is substantially reduced, has stimulated the investigations. Three main nonlinear mechanisms have been invoked.

First, the interaction of sheared flows and in particular zonal $E \times B$ flows with turbulence. Zonal $E \times B$ flows are axisymmetric and poloidally symmetric potential peturbations and their $E \times B$ drift velocity is purely within a magnetic surface. Linearly, they are neither stable nor unstable (at least in the collisionless limit). In a toroidal geometry they may be partly damped because of their coupling to $m \neq 0$ poloidal components, but there remains a totally undamped component. It has been shown in several simulations $[1,3,5,6,9]$ that turbulence generates zonal flows nonlinearly, and that, in turn, the zonal flows reduce the unstable mode amplitudes by shearing the turbulent eddies. Thus, the system appears to be self-regulated, 
with the zonal flows playing the role of 'organizer' of the turbulence. The resulting heat flux is reduced compared with the case when the zonal component is artificially suppressed. Models based on the coupling of turbulence to sheared flows have been proposed [31] to explain the transition from low to high confinement regimes (L-mode to $\mathrm{H}$-mode transition) in the edge region of tokamaks.

Second, intermittency in fluctuations has been observed in some simulations based on fluid [32-37] or collisional gyrokinetic models [38]. This self-organized bursty transport is thought to characterize some regimes of non-diffusive turbulent behaviour. In the context of electron scale instabilities (ETG modes), the estimated linear mixing length for anomalous diffusion is far too low to explain the measured electron transport, essentially because of the very large $k_{\perp}$ of ETG modes. Nonlinear gyrokinetic flux-tube simulations [10] have shown the appearance of intermittent, radially elongated structures, called streamers, that drive (on a time average) much larger fluxes than can be inferred from the linear mixing length estimate. In other simulations, based on a fluid description and a prescribed edge heat flux (as opposed to flux-tube radial periodic boundary conditions), ETG fluctuations do not create the same high level of anomalous heat flux [12]. More investigations are needed in order to resolve this discrepancy. In recent gyrofluid ETG simulations [13] it was shown that zonal flows may be enhanced in weak magnetic shear regions thus leading to an enhanced electron confinement regime.

Third, the mechanism of turbulence spreading has been invoked to explain the saturation of turbulence [39-41]. Already noted in early nonlinear fluid models [42], turbulence is seen propagating radially beyond the region of linear instability. A model equation has been proposed [39] that describes how the radial spreading of the turbulence into the linearly stable zone reduces the intensity of the turbulence in the linearly unstable zone and introduces an additional dependence on the $\rho^{*}=\rho / a$ transport scaling, in broad agreement with toroidal nonlinear gyrokinetic simulations [40]. Saturation occurs when the radial flux of fluctuation energy from the linearly unstable region is balanced by local dissipation in the linearly stable region. In [42], two distinct mechanisms for radial propagation of turbulence are evident: toroidal coupling, acting through convection and nonlinear effects, inducing diffusion. In [41] a model is described in which different drift wave couplings are mediated by zonal flows. In that model, the drift wave radial dispersive properties, which are affected by the toroidal geometry, are an essential feature: the authors expect that turbulence in a torus is qualitatively different from that in a cylinder.

While the above-mentioned models are extremely useful in obtaining an insight into the physics involved in the nonlinear turbulent processes, it is also clear that a more quantitative and detailed description, based on first principles, is desirable. The task can be formulated, in the most simple terms, as one of directly solving the Maxwell-Vlasov system of equations (in the gyrokinetic limit). 'Direct' means here that the first principles that the original system of equations satisfy should be maintained in their numerically discretized version. For example, the particle and energy conservation principles should be reflected in the solution as accurately as possible. Clearly, numerical methods are a necessity. The past decade has seen several major developments in this area [1-16]. Such large-scale simulations have already given important physical results. To cite only a few: the importance of the zonal flows in determining the ITG turbulence level and the corresponding anomalous heat flux [9]; the Shafranov shift stabilization of trapped electron modes in the core of tokamak discharges with internal transport barriers [43]; the scaling of anomalous heat diffusivity with system size [1]; the importance of considering equilibrium distribution functions of the true constants of motion in order to avoid the spurious excitation of geodesic acoustic modes [5,44]; the importance of retaining the parallel nonlinearity for a correct zonal flow determination [6]; detailed comparisons with 
specific tokamak experiments have been made, e.g. with DIII-D [45] and Asdex-Upgrade [46]; the inclusion of non-adiabatic electron response and electromagnetic pertubations in linear [47] and nonlinear [2] global codes; the inclusion of realistic ideal MHD equilibrium geometry and truly global profiles in tokamak [50,46,6], and fully three-dimensional stellarator geometry $[51,52]$ — the latter limited to electrostatic, linear perturbations.

The remainder of the paper is organized as follows. In section 2, the basic features of the gyrokinetic model are introduced, and some of its important variants are mentioned, especially regarding the model used for the electron response. In section 3, the main approaches to the problem are described in brief: Lagrangian versus Eulerian or semi-Lagrangian, global versus local, frozen gradients versus fixed edge flux and free profile evolution. In section 4, we present global gyrokinetic collisionless simulations that are focused on the analysis of the roles of zonal $E \times B$ flows, toroidal geometry and parallel nonlinearity in the development of ITG turbulence in tokamak plasmas. Section 5 presents the main conclusions and discusses possible future work.

\section{Gyrokinetic model and its variations}

We consider a neutral magnetized plasma in a symmetric configuration consisting of nested magnetic surfaces labelled by the variable $\psi$ :

$$
\boldsymbol{B}=F(\psi) \nabla \varphi+\nabla \psi \times \nabla \varphi
$$

in which $F(\psi)$ is the poloidal current flux function, $\varphi$ is the toroidal angle and $\psi$ is the poloidal magnetic flux divided by $2 \pi$, solution of the Grad-Shafranov ideal MHD equilibrium equation [53]. We define the equilibrium scale length of an equilibrium quantity $Q$ as $L_{Q}=|Q / \nabla Q|$. Equilibrium electric fields normal to the magnetic surfaces, $\boldsymbol{E}=-\nabla \Phi_{0}(\psi)$, can be added to the description. Noting the cyclotron frequency of plasma species $\Omega_{\mathrm{s}}$ and their gyroradius $\rho_{\mathrm{L} s}$, we consider low frequency perturbations $\delta \phi$ with the following gyro-ordering in a small parameter $\epsilon_{\mathrm{g}}$ :

$$
\frac{\omega}{\Omega_{\mathrm{i}}} \sim \frac{k_{\|}}{k_{\perp}} \sim \frac{e \delta \phi}{k_{\mathrm{B}} T_{\mathrm{e}}} \sim \frac{\rho_{\mathrm{Li}}}{L_{\mathrm{n}}} \sim \frac{\rho_{\mathrm{Li}}}{L_{\mathrm{Ti}}} \sim \epsilon_{\mathrm{g}}
$$

where $k_{\|}$and $k_{\perp}$ are the components of the wavevector in the directions parallel and perpendicular to the magnetic field $\boldsymbol{B}$. The inhomogeneity of the equilibrium magnetic field is described by another small parameter $\epsilon_{\mathrm{B}} \sim \rho_{\mathrm{Li}} / L_{\mathrm{B}}$. The gyrokinetic ordering removes the fast cyclotron time scale and averaging over the gyroangle reduces the phase space dimension from 6 to 5 [54-57]. The gyrocentre distribution function of species $s$ is $f_{s}\left(\boldsymbol{R}, v_{\|}, \mu\right)$ with $\boldsymbol{R}$ the gyrocentre spatial coordinates, $v_{\|}$the parallel component of the velocity and $\mu=m_{s} v_{\perp}^{2} / 2 B$ the magnetic moment. In collisionless systems we have

$$
f_{s}=\text { constant }
$$

along the characteristics, which are solutions of the following evolution equations of motion

$$
\begin{aligned}
& \frac{\mathrm{d} \mu}{\mathrm{d} t}=0, \\
& \frac{\mathrm{d} \boldsymbol{R}}{\mathrm{d} t}=\left.\frac{\mathrm{d} \boldsymbol{R}}{\mathrm{d} t}\right|_{\|}+\left.\frac{\mathrm{d} \boldsymbol{R}}{\mathrm{d} t}\right|_{\nabla B}+\left.\frac{\mathrm{d} \boldsymbol{R}}{\mathrm{d} t}\right|_{E}+\left.\frac{\mathrm{d} \boldsymbol{R}}{\mathrm{d} t}\right|_{\delta E}, \\
& \frac{\mathrm{d} v_{\|}}{\mathrm{d} t}=\left.\frac{\mathrm{d} v_{\|}}{\mathrm{d} t}\right|_{\nabla B}+\left.\frac{\mathrm{d} v_{\|}}{\mathrm{d} t}\right|_{E}+\left.\frac{\mathrm{d} v_{\|}}{\mathrm{d} t}\right|_{\delta E} .
\end{aligned}
$$


Explicit expressions for the terms appearing on the right-hand side can be found in [57]. The subscripts $\|, \nabla B, E$ and $\delta E$ denote parallel motion, perpendicular magnetic drifts, equilibrium $E \times B$ drifts and perturbed $\delta E \times B$ drifts, respectively. Due to the gyrokinetic ordering, the parallel time derivative is larger than the other terms. This is the origin of the strong anisotropy of the system of perturbations, which tend to form structures aligned with the equilibrium magnetic field lines. This strong anisotropy, and the associated separation of parallel and perpendicular time scales, are essential features of micro-instabilities and turbulence in this frequency range. The system of equations is completed by Poisson's equation for $\delta \phi$, which reduces to the quasi-neutrality equation

$$
\delta n_{\mathrm{e}}-\delta n_{\mathrm{i}, \mathrm{pol}}=\left\langle\delta n_{\mathrm{i}}\right\rangle,
$$

where $\delta n_{\mathrm{i}, \text { pol }}$ is the perturbed ion polarization density, $\left\langle\delta n_{\mathrm{i}}\right\rangle$ is the perturbed gyro-averaged gyrocentre ion density and $\delta n_{\mathrm{e}}$ is the perturbed electron density.

The simplest model for electrons, applied to the study of ITG modes, assumes an adiabatic (or Boltzmann) electron response in the direction parallel to the magnetic field lines. The justification is that parallel electron motion is so fast that the electron population can establish a Boltzmann equilibrium at all times. This is equivalent to neglecting the electron inertia $\left(m_{\mathrm{e}} \rightarrow 0\right)$ for the parallel electron motion. We obtain then $e \nabla_{\|} \phi-\left(k_{\mathrm{B}} T_{\mathrm{e}} / n_{\mathrm{e}}\right) \nabla_{\|} n_{\mathrm{e}}=0$, the solution of which is $n_{\mathrm{e}}=\mathcal{N}(s) \exp \left[e \phi / k_{\mathrm{B}} T_{\mathrm{e}}\right]$, where $s$ is a label of the magnetic field line. The normalization constant $\mathcal{N}$ can be fixed in configurations with nested flux surfaces by noting that the number of electrons should be conserved on flux surfaces. We obtain

$$
\delta n_{\mathrm{e}}=\frac{e n_{0}}{k_{\mathrm{B}} T_{\mathrm{e}}}(\delta \phi-\overline{\delta \phi}), \quad \overline{\delta \phi}=\frac{\int_{\psi} \delta \phi(s, \theta, \varphi) J(s, \theta) \mathrm{d} \theta \mathrm{d} \varphi}{\int_{\psi} J(s, \theta) \mathrm{d} \theta \mathrm{d} \varphi},
$$

where $J=(\nabla s \cdot \nabla \theta \times \nabla \varphi)^{-1}$ is the Jacobian. The system of equations is (3)-(8). Note that the nonlinearity of the system appears in the last terms of equation (5), the perpendicular nonlinearity, and of equation (6), the parallel nonlinearity.

For the study of TEM, it is appropriate to consider adiabatic passing electrons and driftkinetic trapped electrons:

$$
\delta n_{\mathrm{e}}=\frac{e n_{0}}{k_{\mathrm{B}} T_{\mathrm{e}}}\left(1-\alpha_{b}\right)(\delta \phi-\overline{\delta \phi})+\left.\delta n_{\mathrm{e}}\right|_{\text {trapped }},
$$

where $\alpha_{b}$ is the fraction of trapped electrons.

For the study of electromagnetic perturbations, passing electrons must also be considered as non-adiabatic, and the system of equations is to be completed by Ampère's law. In addition to the ITG and TEM modes, the model describes another branch of micro-instabilities, named Alfvénic-ITG [58] or kinetic ballooning mode [59]. For finite but small $\beta$, it is sufficient to consider a two-potential approach $\left(\phi, A_{\|}\right)$which neglects the perturbations of the magnetic field parallel to the equilibrium $\left(\delta B_{\|} \approx 0\right)$. A full electromagnetic model in tokamak geometry taking into account finite $\delta B_{\|}$formulated with a spectral approach can be found in [47].

Collisionality enters mainly in two different mechanisms. Electron-ion pitch angle collisions cause trapping/detrapping of electrons and therefore affect TEM. Ion-ion collisions cause damping of zonal flows and this results in an increase of the ITG turbulence level. This mechanism was studied in [48] with a three-dimensional fluid model; results show an energy confinement time increase with decreasing collisionality and are interpreted as an increase in the effective critical temperature gradient due to the increased zonal flow amplitude, the so-called 'upshift' first noticed in [3].

Finally, we should mention the gyro-Landau fluid equations which consist of a set of moments of the gyrokinetic equations that include models of the kinetic resonances such as Landau damping [60], linear and nonlinear finite Larmor radius effects [61] and toroidal 
drift [62]. An alternative non-dissipative closure model was introduced in [63]. Nonlinear gyrofluid simulations showed, for the first time, the importance of zonal flows in the saturation of ITG turbulence. Zonal flows have a linearly completely undamped component [64] and the closure of gyrofluid equations should reflect this property.

\section{Numerical approaches}

Two classes of approach have been used for the numerical resolution of gyrokinetic equations. The Lagrangian approach consists of a pseudo-random sampling of $f$ over phase space at points called 'markers' or 'tracers', an operation called 'loading'. Then their orbits are followed according to equations (5) and (6), the so-called 'pushing'. One could assimilate this procedure to a completely unstructured, moving grid. At every time step the density in real space is reconstructed by a projection of the markers, an operation called 'charge assignment'. The field equations are then solved on a fixed grid in real space, e.g. with a finite element technique [50]. The advantages of this particle-in-cell (PIC) technique are its conceptual simplicity and the ability to integrate systems of high dimensionality. The main drawback is the problem of statistical noise inherent to this approach and the associated slow convergence rate $\sim 1 / \sqrt{N}$ with the number of markers. Noise can build up and accumulate in nonlinear simulations to a point where the basic physical properties of the system, such as charge and energy conservation, are violated. A method to reduce this problem was formulated some time ago, the $\delta f$ approach, which statistically samples the perturbed part of the distribution function only [65]. A further improvement consists of choosing an appropriate distribution of the markers in phase space ('optimized loading'). It was shown in [4] that the application of this technique leads to a substantial improvement in the quality of the numerical simulation as measured by the verification of the energy conservation property. It was shown in [6] that this is crucial in order to obtain a physically correct level of zonal flows, turbulence and anomalous heat flux. A further simplification was achieved by showing that the integration of the time evolution equation for $\delta f$ along the perturbed characteristics is actually redundant, and it is perfectly appropriate simply to take $\delta f=\left.f\left(\boldsymbol{R}, v_{\|}, \mu\right)\right|_{t=0}-\left.f_{0}\left(\boldsymbol{R}, v_{\|}, \mu\right)\right|_{t=t}$, where $f_{0}$ is a known distribution function [66]. Moreover, the time evolution equation for $\delta f$ assumes that $f_{0}$ is a constant along the unperturbed orbits (in other words it is an equilibrium distribution function) therefore it should be a function of the constants of motion of the unperturbed orbits: particle energy $\mathcal{E}$, magnetic moment $\mu$ and conjugate toroidal momentum $\psi_{0}=\psi+\left(m_{s} / q_{s}\right)(F / B) v_{\|}$. If a local approximation is chosen for $f_{0}$, i.e. a function of $\psi$ instead of $\psi_{0}$, this can lead to a spurious drive of zonal flows [5].

The Eulerian approach (sometimes called 'Vlasov' in the literature) [67] consists of discretizing the phase space on a fixed grid. The time evolution equations for $f$ are solved using finite difference or finite element techniques. The time integration can be performed using explicit or semi-implicit schemes. An advanced code using this approach in the context of gyrokinetic simulations is described in detail in [2]. An advantage of the Eulerian approach (compared to the Lagrangian one) is that it is, in principle, easier to control the phase space gridding. In addition, the numerical properties of convergence with mesh size should be free of statistical noise. The drawbacks are perhaps that this approach is technically more difficult to implement, and that the appearance of fine scale structures in phase space ('filamentation') requires very fine grid resolution in certain regions of phase space. An intermediate approach, called semi-Lagrangian or semi-Eulerian, consists of discretizing the phase space on a fixed grid, as in the Eulerian scheme, and following orbits in phase space, as in the Lagrangian scheme, with $f$ interpolated from the previous time step at the foot of the orbit [68]. 
Numerical approaches differ in the level of globality that the schemes can support. From local to global we find in increasing order: (a) the ballooning approximation, that solves along a field line and assumes large toroidal mode numbers; (b) the flux-tube geometry that solves in a finite size domain in the vicinity of a field line and assumes periodic boundary conditions; (c) the toroidal ring in which a finite size radial slice of the plasma is simulated; (d) the full radius approach in which the equations are solved from the magnetic axis to the plasma boundary. The most global approach is of course highly desirable and even necessary when structures develop with a radial extent comparable to the system size. The flux-tube and toroidal ring approaches certainly make sense when studying electron scale turbulence for which a full radius scheme would be prohibitive. The drawback of the flux-tube approach is in its unphysical radial periodic boundary conditions. The toroidal ring approach allows for more physical boundary conditions such as fixed edge flux and evolving temperature profiles. There are more variations in the way the time evolution of plasma profiles is considered. In several codes a fixed temperature gradient profile is assumed, sources and sinks may be added, or a dissipative term is introduced that models relaxation to an initial Maxwellian. In other codes the temperature profiles are defined consistently with the temperature gradient profiles and, moreover, are left free to evolve in time.

The difficulty associated with the large parallel/perpendicular anisotropy in time and length scales has prompted the development of field-aligned meshes [1,2]. Another approach is to define a magnetic straight-field line coordinate and apply a variable transformation to extract the fast poloidal phase variation: a gain in code performance of one or two orders of magnitude can be reached $[5,50]$.

The validation of numerical simulation codes is extremely important considering the complexity of the physics involved. This requires not only case comparisons with selected experiments $[45,46]$ but also a verification of the first principles these codes are based on $[4,6]$. Another important element is to perform cross benchmarking of the different codes [3].

\section{Toroidal coupling, zonal flows and parallel nonlinearity}

Toroidal coupling enters at three different points in the gyrokinetic equations. First, the particle motion grad-B drifts have the effects of (a) driving a toroidally coupled mode (e.g. the toroidalITG) more unstable than its 'slab' counterpart and (b) creating a trapped particle population in the minimum B regions which can cause other modes to become unstable (TEM and TIM). Second, the geometry appears explicitly in the field equations through the expressions of differential operators. Third, in the adiabatic electron response, the zonal component, $\overline{\delta \phi}$, contains a Jacobian of the toroidal magnetic coordinates, see equation (8).

We consider a tokamak configuration given by an ideal MHD equilibrium with the following parameters: aspect ratio $R / a=2.2$, minor radius $a=96 \rho_{s}, q_{0}=1.7, q_{a}=4.7, T_{i}$ profile with $\max \left(R / L_{T}\right)=7.9$ (which is about twice as high as the linear instability threshold for toroidal ITG modes), uniform density and $T_{\mathrm{e}}$ profiles. We solve the nonlinear electrostatic gyrokinetic equations (3)-(8) with the full radius code ORB5 [69] using $N=33 \times 10^{6}$ markers, and cubic spline finite elements in $(s, \theta, \varphi)$ on a $32 \times 256 \times 128$ grid. All profiles are global and free to evolve. The model includes parallel nonlinearity and toroidal coupling in the zonal component, equation (8). The initial distribution function $f$ is a canonical Maxwellian, i.e. function of the constants of motion of the unperturbed orbits. Figure 1 shows the time traces of field energy and heat flux and figure 2 radial profiles of the zonal $E \times B$ velocity and effective temperature at different time slices. While in the early stages of the nonlinear process small-scale zonal flows appear, at a later stage the radial structure of zonal flows expands radially to form finally two very broad radial zones. Global simulations in a cylinder $[6,70,71]$ 

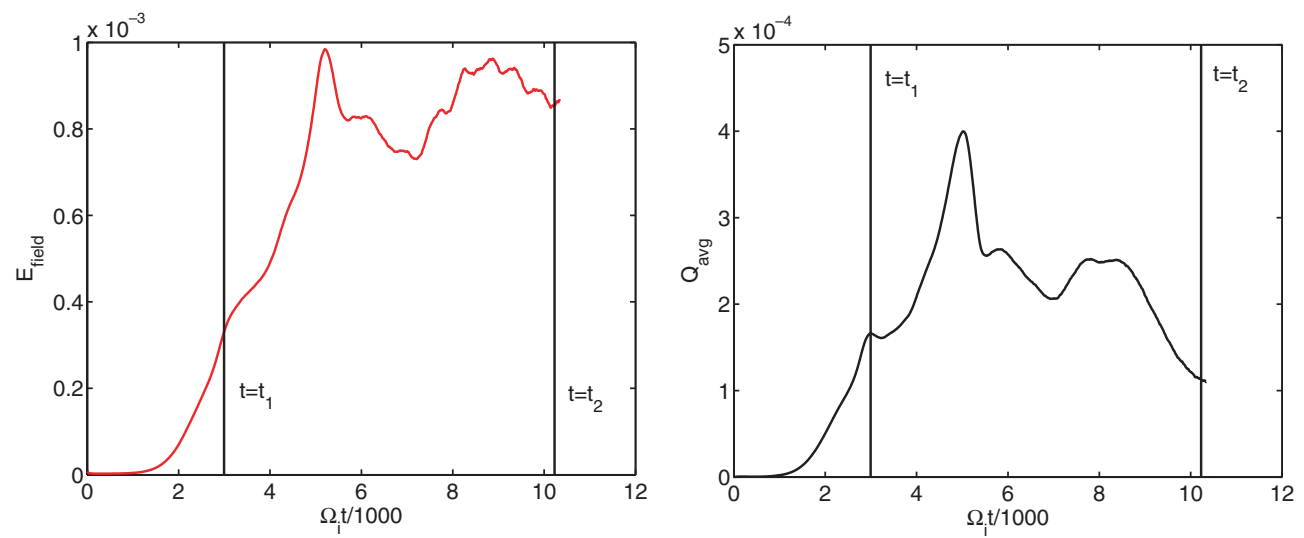

Figure 1. Time evolution of field energy (left) and averaged heat flux (right) in a tokamak of aspect ratio 2.2. The simulation includes the toroidal coupling of zonal flows coming through the adiabatic electron response, equation (8).
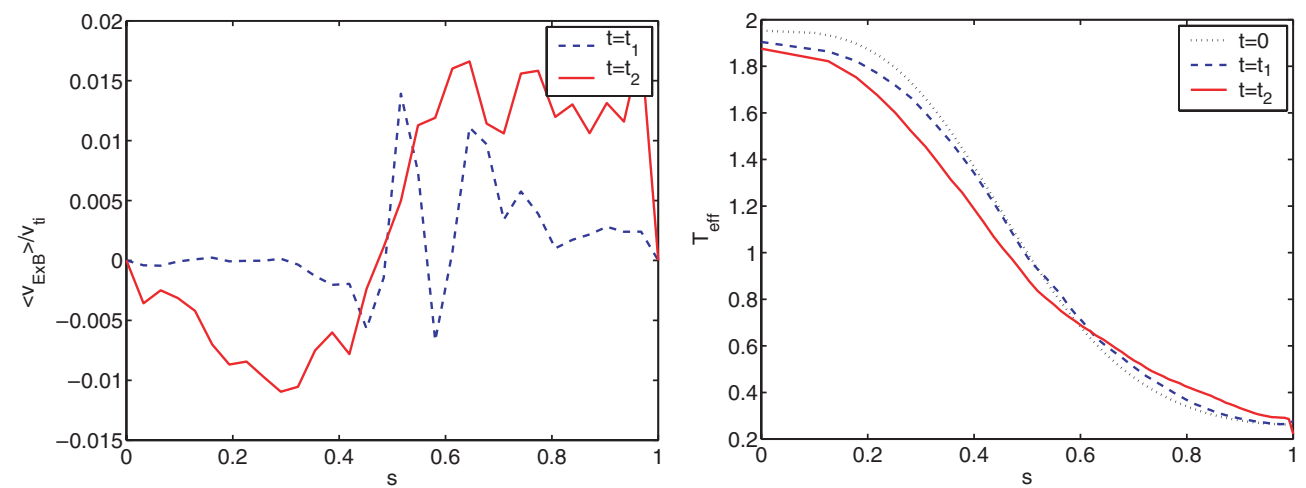

Figure 2. Radial profiles of magnetic surface averaged zonal $E \times B$ velocity (left) and effective temperature profiles taken in the early $\left(---, t=t_{1}\right)$ and late $\left(-, t=t_{2}\right)$ nonlinear phases, for the simulation of figure 1 .

had shown a persistent radial structure of zonal flows with a much finer radial structure. The explanation for this different behaviour may lie in the evolution of the effective temperature profile (figure 2): for the toroidal case the instability drive is much stronger and consequently the profile evolution occurs on a much shorter time scale compared to the cylindrical case: the effective temperature profile evolves quickly to a profile which is closer to the marginal stability of the toroidal-ITG mode.

The above results have been obtained with the parallel nonlinearity retained (last term in equation (6)). A comparison between two simulations, with and without parallel nonlinearity, is shown in figure 3, for the same toroidal case as in figures 1 and 2, and in which the optimized loading scheme [4] has been applied in order to reduce the statistical noise accumulation at long simulation times. There is a visible effect on the zonal $E \times B$ flow pattern. Note that an effect of the parallel nonlinearity on zonal $E \times B$ flow structures was already found in cylindrical simulations $[6,70,71]$.

The evolution of zonal flows towards broad radial structures is confirmed in computations using the nonlinear global gyrokinetic code GT3D [5] that includes, as for the ORB5 code, 


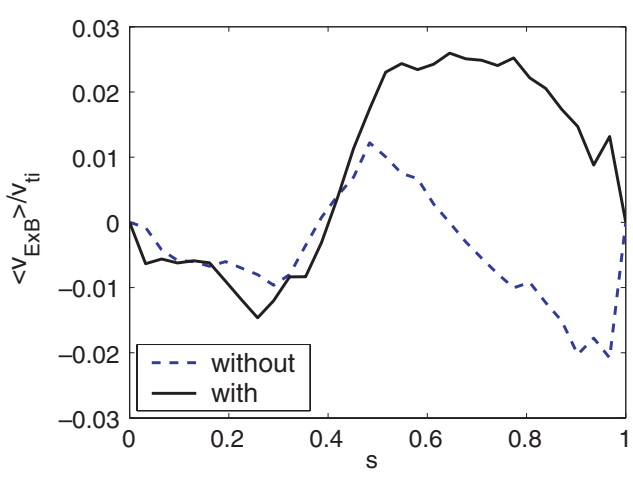

Figure 3. Radial profiles of magnetic surface averaged zonal $E \times B$ velocity in the late nonlinear phase, for simulations with (- ${ }^{-}$) and without (- - -) parallel nonlinearity. Same physical parameters as for figures 1 and 2, optimized loading applied.
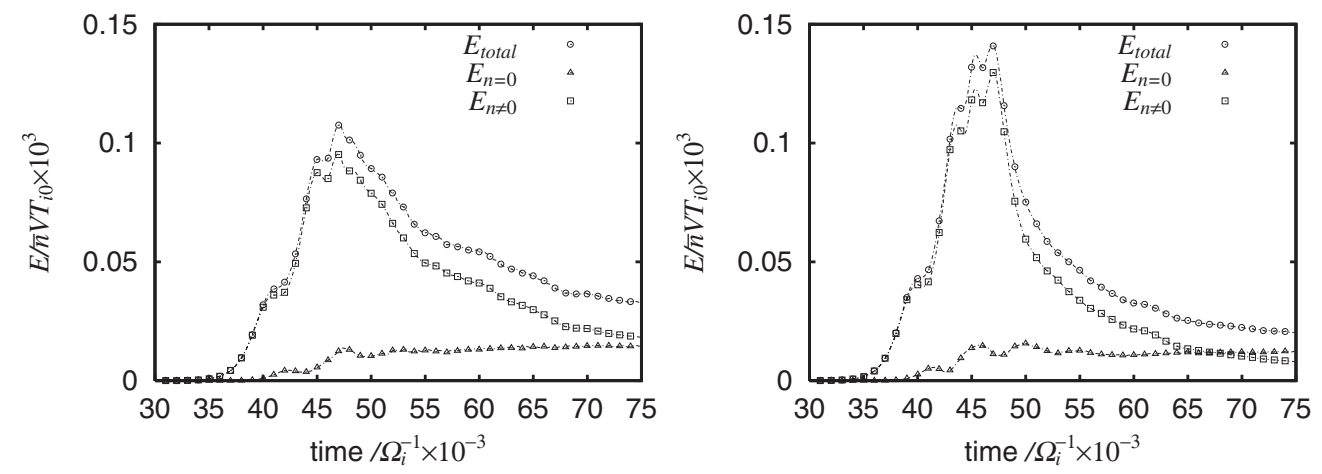

Figure 4. Time evolution of perturbed field energy, total (circles), in axisymmetric (triangles) and non-axisymmetric (squares) modes; without (left) and with (right) toroidal coupling in the surface averaged zonal component $\overline{\delta \phi}$, equation (8). Cyclone base case parameters.

parallel nonlinearity and a canonical Maxwellian. The perturbed field is represented on twodimensional quadratic spline finite elements with 76 non-uniform radial intervals, 32 poloidal intervals with a quasi-ballooning representation [50] and 32 toroidal Fourier components. We use parameters of the Cyclone base case [3]: $R / a=2.7, a=150 \rho_{\mathrm{Li}}, R / L_{T}=6.9$. Two series of simulations are performed, one using the cylindrical approximation for the zonal component, $\overline{\delta \phi} \approx \delta \phi_{m=0, n=0}$, the other with the true toroidal Jacobian, equation (8). The optimized loading technique [4] is applied in order to reduce numerical noise and 160 million tracers are used. Energy conservation is better than $30 \%$ of the perturbed field energy for the cases shown in figures 4 and 5. The time evolution of the field energy, figure 4, shows remarkable differences: in the simulation with approximated zonal flows (left), the peak in field energy is about 30\% lower. After the peak, the decay time is much longer, resulting in a twice higher field energy in non-axisymmetric modes at the end of the simulation, as compared to the case with true toroidal zonal flows (right). The remaining slow decay is due to a slow temperature gradient flattening (note that there are no sources in our simulations). We show in figure 5 (left) a contour plot of the zonal $E \times B$ velocity versus minor radius and time, for the case with toroidal zonal flows. The appearance of broad zones (three in this case) at later stages is very clear. The small amplitude oscillations near the plasma edge are 

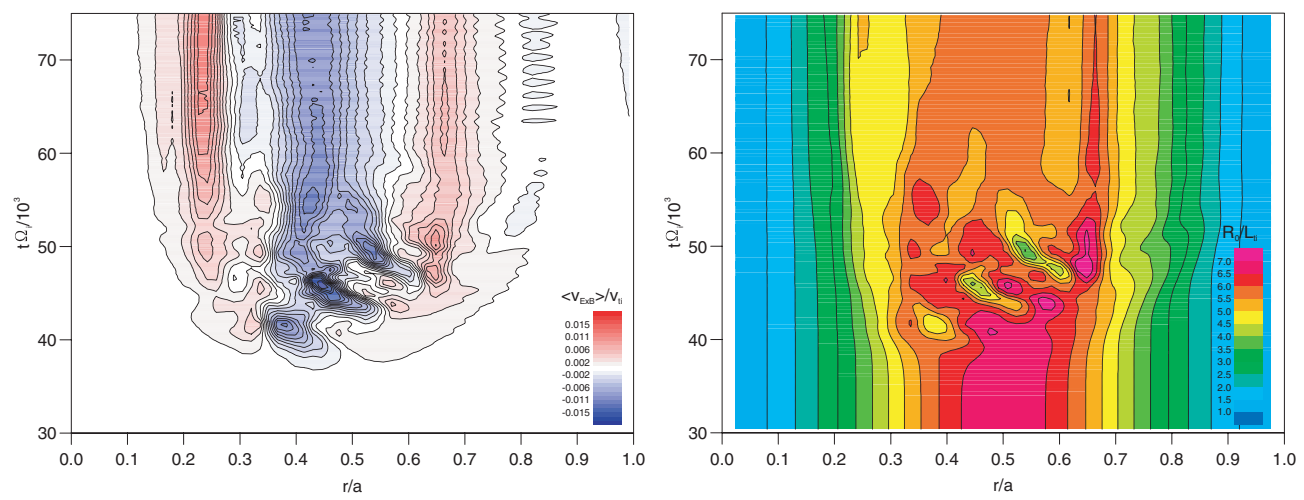

Figure 5. Left: contours of flux averaged $E \times B$ drift velocity versus $r / a$ and $t$. Colour scale: blue for negative, red for positive, $\min / \max :\left\langle v_{E} \times B\right\rangle= \pm 0.018 v_{\mathrm{ti}}$. Right: contours of effective ion temperature gradient inverse scale length. Colour scale: $\max R / L_{T}=7$.

(This figure is in colour only in the electronic version)

geodesic acoustic modes (GAM) [44]. In the earlier stages, a series of peaks in the $E \times B$ velocity appears at increasing radial positions. These peaks immediately follow bursts in heat flux which in turn cause local and temporary flattenings of the temperature gradient (figure 5, right) at the corresponding locations. The fast time scale radial turbulence spreading is thus caused by avalanche processes, with changes in temperature gradient due to bursts in heat flux and excitation of ITG fluctuations followed by zonal flow bursts. The temperature gradient long time evolution shows a visible flattening, bringing it closer to the linear marginal stability threshold of $R / L_{T} \approx 5$ (computed in the absence of $E \times B$ flows).

\section{Conclusions and future developments}

In both cylindrical $[6,70,71]$ and toroidal simulations presented here, radial turbulence spreading is observed. For both cases zonal flows organize the turbulence and the system evolves to a quasi-steady state with a regular pattern of zonal $E \times B$ flows. For both cases it is important to retain the parallel nonlinearity in order to obtain a correct zonal $E \times B$ flow structure. In a torus, the stronger instability drive is such that the nonlinearity due to profile evolution becomes important. Toroidal coupling of zonal flows is affecting their interaction with ITG driven perturbations. This has a measurable effect on their development and saturation. In a torus, fast radial turbulence spreading is caused by sequences of avalanches in which bursts of ITG turbulence are accompanied by temperature gradient local flattening and followed by zonal $E \times B$ bursts. The system evolves in the long term to a state characterized by quasi-steady zonal structures of a much larger width $\left(30-50 \rho_{\mathrm{Li}}\right)$ than in a cylinder $\left(\sim 10 \rho_{\mathrm{Li}}\right)$. This difference may be related to the radially more extended structures of the toroidal ITG mode compared to the 'slab' ITG mode, although more extensive studies are needed to confirm this.

There are still challenges for future numerical simulations. Generally speaking, electron transport and density transport are much less well understood. Another feature observed in experiments, namely the relation of rational $q_{\mathrm{min}}$ values in reversed shear discharges with the appearance of internal transport barriers, has not been fully understood as yet. Another open question is the finite $\beta$ dependence of anomalous transport. All these questions will require consideration of non-adiabatic electron response and even electromagnetic perturbations. 
While numerical tools have recently been developed for these purposes, there remains considerable work in order to answer these questions with sufficiently accurate first principles based simulations.

\section{Acknowledgments}

We wish to thank J Nührenberg, Y Kishimoto, P H Diamond, T S Hahm and Z Lin for stimulating discussions. The work was partly supported by the Swiss National Science Foundation. Computations were performed on the JAERI Origin 3800 system and on the EPFL Pleiades cluster.

\section{References}

[1] Lin Z, Hahm T S, Lee W W, Tang W M and White R B 1998 Science 2811835

[2] Candy J and Waltz R E, 2003 J. Comput. Phys. 186545

[3] Dimits A M et al 2000 Phys. Plasmas 7969

[4] Hatzky R et al 2002 Phys. Plasmas 9898

[5] Idomura Y, Tokuda S and Kishimoto Y 2003 Nucl. Fusion 43234

[6] Villard L et al 2004 Nucl. Fusion 44172

[7] Parker S E, Chen Y, Wan W, Cohen B I and Nevins W M 2004 Phys. Plasmas 112594

[8] Scott B D 2002 New J. Phys. 452

[9] Sydora R D, Decyck V K and Dawson J M 1996 Plasma Phys. Control. Fusion 38 A281

[10] Jenko F, Dorland W, Kotschenreuther M and Rogers B N 2000 Phys. Plasmas 71904

[11] Dorland W, Jenko F, Kotschenreuther M and Rogers B N 2000 Phys. Rev. Lett. 855579

[12] Labit B and Ottaviani M 2003 Phys. Plasmas 10126

[13] Li J Q and Kishimoto Y 2004 Phys. Plasmas 111493

[14] Dimits A M and Lee W W 1993 J. Comput. Phys. 107309

[15] Manuilskiy I and Lee W W 2000 Phys. Plasmas 71381

[16] Watanabe T H and Sugama H 2004 Phys. Plasmas 111476

[17] Degtyarev L et al 1997 Comput. Phys. Commun. 10310

[18] Angioni C, Goodman T P, Henderson M A and Sauter O 2003 Nucl. Fusion 43455

[19] Miller R L et al 1997 Phys. Plasmas 41062

[20] Cooper W A et al 2002 Plasma Phys. Control. Fusion 44 B357

[21] Lütjens H and Luciani J F 2002 Phys. Plasmas 94837

[22] Fu G Y and Van Dam J W 1989 Phys. Fluids B 11949

[23] Cheng C Z 1991 Phys. Fluids B 32463

[24] Villard L, Brunner S and Vaclavik J 1995 Nucl. Fusion 351173

[25] Jaun A, Fasoli A, Vaclavik J and Villard L 2000 Nucl. Fusion 401343

[26] Guzdar P N, Chen L, Tang W M and Rutheford P H 1983 Phys. Fluids 26673

[27] Adam J C, Tang W M and Rutheford P H 1976 Phys. Fluids 19561

[28] Horton W, Hong B G and Tang W M 1988 Phys. Fluids 312971

[29] Gladd N T, Drake J F, Chang C L and Lin C S 1980 Phys. Fluids 231182

[30] Connor J W, Fukuda T, Garbet X, Gormezano C, Mukhovatov V and Wakatani M 2004 Nucl. Fusion 44 R1

[31] Diamond P H, Liang Y-M, Carreras B A and Terry P W 1994 Phys. Rev. Lett. 722565

[32] Garbet X et al 1999 Nucl. Fusion 392063

[33] Sarazin Y, Garbet X, Ghendrih P and Benkadda S 2000 Phys. Plasmas 71085

[34] Benkadda S et al 2001 Nucl. Fusion 41995

[35] Graves J P, Dendy R O, Hopcraft K I and Jakeman E 2002 Phys. Plasmas 91596

[36] Tangri V, Das A, Kaw P and Singh R 2003 Phys. Rev. Lett. 91025001

[37] Li J and Kishimoto Y 2002 Phys. Rev. Lett. 89115002

[38] Lin Z, Hahm T S, Lee W W, Tang W M and White R B 2000 Phys. Plasmas 71857

[39] Hahm T S et al 2004 Plasma Phys. Control. Fusion 46 A323

[40] Lin Z and Hahm T S 2004 Phys. Plasmas 111099

[41] Zonca F, White R B and Chen L 2004 Phys. Plasmas 112488

[42] Garbet X, Laurent L, Samain A and Chinardet J 1994 Nucl. Fusion 34963

[43] Beer M A et al 1997 Phys. Plasmas 41792 
[44] Winsor N, Johnson J L and Dawson J M 1968 Phys. Fluids 112448

[45] Candy J and Waltz R E 2003 Phys. Rev. Lett. 91045001

[46] Bottino A, Peeters A G, Sauter O, Vaclavik J and Villard L 2004 Phys. Plasmas 11198

[47] Ganesh R, Angelino P, Vaclavik J and Villard L 2004 Phys. Plasmas 113106

[48] Falchetto G L and Ottaviani M 2004 Phys. Rev. Lett. 92025002

[49] Chen Y and Parker S E 2003 J. Comput. Phys. 189463

[50] Fivaz M et al 1998 Comput. Phys. Commun. 11127

[51] Jost G, Tran T M, Cooper W A, Villard L and Appert K 2001 Phys. Plasmas 83321

[52] Kornilov V, Kleiber R, Hatzky R, Villard L and Jost G 2004 Phys. Plasmas 113196

[53] Freidberg J P 1987 Ideal Magnetohydrodynamics (New York: Plenum)

[54] Dubin D H E, Krommes J A, Oberman C and Lee W W 1983 Phys. Fluids 263524

[55] Hahm T S 1988 Phys. Fluids 312670

[56] Brizard A 1989 J. Plasma Phys. 41541

[57] Hahm T S 1996 Phys. Plasmas 34658

[58] Zonca F, Chen L, Dong J Q and Santoro R A 1999 Phys. Plasmas 61917

[59] Kim J Y, Horton W and Dong J Q 1993 Phys. Fluids B 54030

[60] Hammett G W and Perkins F W 1990 Phys. Rev. Lett. 643019

[61] Dorland W and Hammett G W 1993 Phys. Fluids B 5812

[62] Waltz R E, Dominguez R R and Hammett G W 1992 Phys. Fluids B 43138

[63] Sugama H, Watanabe T H and Horton W 2003 Phys. Plasmas 10726

[64] Rosenbluth M N and Hinton F L 1998 Phys. Rev. Lett. 80724

[65] Parker S E and Lee W W 1993 Phys. Fluids B 577

[66] Allfrey S J and Hatzky R 2003 Comput. Phys. Commun. 15498

[67] Manfredi G, Shoucri M, Dendy R O, Ghizzo A and Bertrand P 1996 Phys. Plasmas 3202

[68] Sonnendrucker E, Roche J, Bertrand P and Ghizzo A 1999 J. Comput. Phys. 149201

[69] Tran T M et al 1998 Proc. Int. Workshop (Varenna) Theory of Fusion Plasmas (Bologna, Italy: Editrice Compositori) $\mathrm{p} 45$

[70] Allfrey S J et al 2002 New J. Phys. 4 art.no.29

[71] Allfrey S J et al 2002 Proc. Int. Workshop (Varenna) Theory of Fusion Plasmas (Bologna, Italy: Società Italiana di Fisica) p 171 